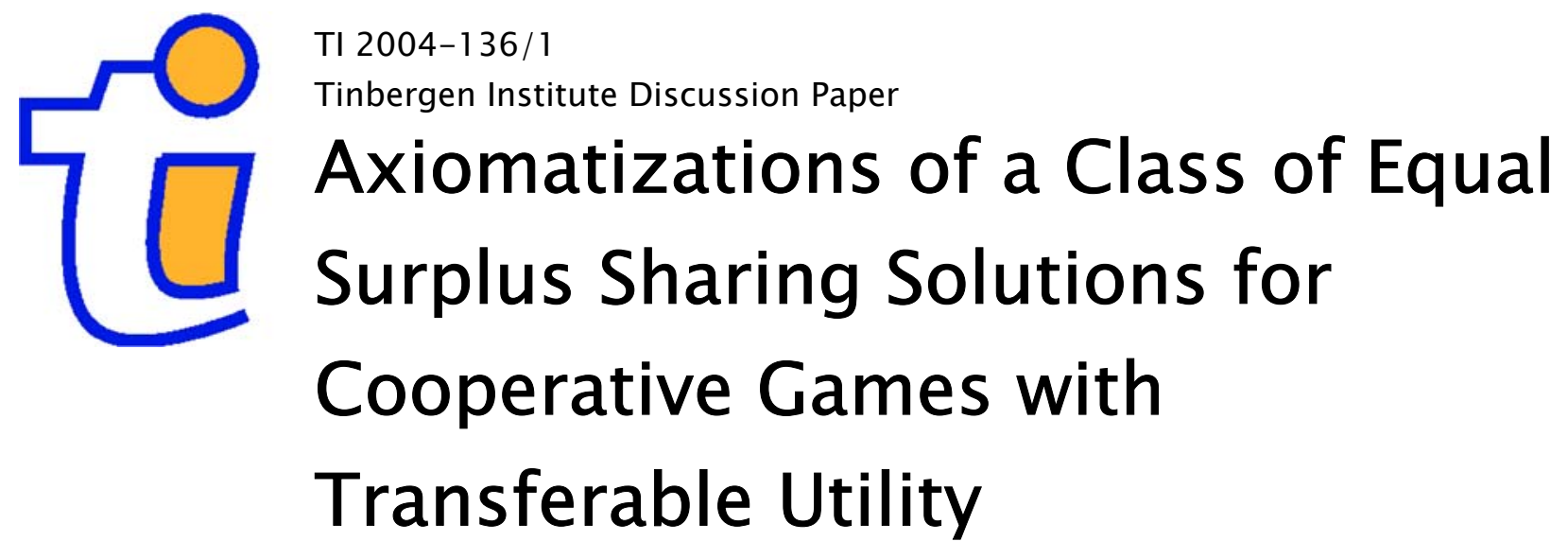

René van den Brink'

Yukihiko Funaki2

${ }^{\prime}$ Faculty of Economics and Business Administration, Vrije Universiteit Amsterdam, and Tinbergen Institute,

2 School of Political Science and Economics, Waseda University, Tokyo. 
Tinbergen Institute

The Tinbergen Institute is the institute for economic research of the Erasmus Universiteit Rotterdam, Universiteit van Amsterdam, and Vrije Universiteit Amsterdam.

Tinbergen Institute Amsterdam

Roetersstraat 31

1018 WB Amsterdam

The Netherlands

Tel.: $\quad+31(0) 205513500$

Fax: $\quad+31(0) 205513555$

Tinbergen Institute Rotterdam

Burg. Oudlaan 50

3062 PA Amsterdam

The Netherlands

Tel.: $\quad+31(0) 104088900$

Fax: $\quad+31(0) 104089031$

Please send questions and/or remarks of nonscientific nature to driessen@tinbergen.nl.

Most TI discussion papers can be downloaded at http://www.tinbergen.nl. 


\title{
Axiomatizations of a Class of Equal Surplus Sharing Solutions for Cooperative Games with Transferable Utility
}

\author{
René van den Brink* Yukihiko Funaki ${ }^{\dagger}$
}

December 16, 2004

*Department of Econometrics and Tinbergen Institute, Free University, De Boelelaan 1105, $1081 \mathrm{HV}$ Amsterdam, The Netherlands. E-mail: jrbrink@feweb.vu.nl

${ }^{\dagger}$ Department of Economics, School of Political Science and Economics, Waseda University, 1-6-1 Nishi-Waseda, Shinjuku-Ku, Tokyo, 169-8050 Japan. E-mail: funaki@waseda.jp 


\begin{abstract}
A situation in which a finite set of players can obtain certain payoffs by cooperation can be described by a cooperative game with transferable utility, or simply a TU-game. A onepoint solution for TU-games assigns a payoff distribution to every TU-game. In this paper we discuss a class of solutions containing all convex combinations of the CIS-value, the ENSC-value, and the egalitarian solution. We characterize this class of solutions using a general standardness for two player games and reduced game consistency. Specific cases of these properties characterize specific solutions in this class.
\end{abstract}

Keywords: TU-game, Equal surplus sharing, CIS-value, ENSC-value, Egalitarian solution, Reduced Game Consistency

JEL code: C71

AMS subject classification: $91 \mathrm{~A} 12$ 


\section{Introduction}

A situation in which a finite set of players can obtain certain payoffs by cooperation can be described by a cooperative game with transferable utility, or simply a TU-game, being a pair $(N, v)$, where $N \subset \mathbb{N}$ is a finite set of players with $|N| \geq 2$ and $v: 2^{N} \rightarrow \mathbb{R}$ is a characteristic function on $N$ such that $v(\emptyset)=0$. For any coalition $S \subseteq N, v(S)$ is called the worth of coalition $S$. This is what the members of coalition $S$ can obtain by agreeing to cooperate. We denote the class of all TU-games by $\mathcal{G}$.

A payoff vector of game $(N, v)$ is an $|N|$-dimensional real vector $x \in \mathbb{R}^{n}, n=|N|$, which represents a distribution of the payoffs that can be earned by cooperation over the individual players. A (point-valued) solution for TU-games is a function $\psi$ which assigns a payoff vector $\psi(N, v)$ to every TU-game $(N, v)$. If a solution assigns to every game a payoff vector that exactly distributes the worth of the 'grand coalition' $N$ then the solution is called efficient ${ }^{1}$.

In this paper we discuss a class of solutions for TU-games that all have some egalitarian flavour in the sense that they assign to every player some initial payoff and distribute the remainder of $v(N)$ equally among all players. Examples of such solutions are the CIS-value, the ENSC-value and the egalitarian solution. The Center-of-gravity of the Imputation-Set value, shortly denoted by CIS-value (see Driessen and Funaki (1991)), assigns to every player its individual worth, and distributes the remainder of the worth of the grand coalition $N$ equally among all players. The Egalitarian Non-Separable Contribution value, shortly denoted by ENSC-value, assigns to every game $(N, v)$ the CIS-value of its dual game. The egalitarian solution just distributes the worth of the 'grand coalition' equally among all players.

In this paper we define a class of solutions that contains the above mentioned solutions and all convex combinations of these solutions. We begin by defining this class for two-player games. For this class of games our solutions are defined by a weak standardness for two-player games. The usual standardness for two-player games states that in a two player game every player earns its own worth plus half of what remains of the worth of the two-player ('grand') coalition (see, e.g. Hart and Mas-Colell $(1988,1989)$ ). The weaker standardness that we discuss also contains egalitarian standardness for two-player games. We also discuss some properties, in particular properties that characterize efficient, symmetric and anonymous solutions for two-player games.

After defining the class of solutions for two-player games we extend the definition to $n$-player games, by applying some reduced game consistency. We also discuss some other properties of the class of solutions obtained in this way. After that we provide some

\footnotetext{
${ }^{1}$ Efficient solutions are often called values.
} 
axiomatic characterizations of specific solutions in this class, in particular the CIS-value, the ENSC-value, their average and the egalitarian solution. Besides characterizations that follow as corollaries from our main theorem using weak standardness for two-player games and reduced game consistency, we discuss characterizations using self-duality and weak individual rationality, and characterizations as compromise values.

The paper is organized as follows. Section 2 discusses some preliminaries on TU-games and solutions. In Section 3 we define our class of solutions for two-player games. In Section 4 we extend this definition to $n$-player games using a reduced game consistency. In Section 5 we discuss some specific examples of solutions that are obtained in this way, and give axiomatic characterizations of these examples. In Section 6 we discuss axiomatic characterizations of the class and some specific solutions using weak individual rationality. In Section 7 we look at the solutions as compromise values. Section 8 contains some concluding remarks. Finally, there is an appendix that discusses the reduced games.

\section{Preliminaries}

A TU-game $(N, v)$ is monotone if $v(S) \leq v(T)$ for all $S \subseteq T \subseteq N$. A TU-game $(N, v)$ is non-negative if $v(S) \geq 0$ for all $S \subseteq N$. A TU-game $(N, v)$ is weakly essential if $\sum_{i \in N} v(\{i\}) \leq v(N)$. A TU-game $(N, v)$ is convex if $v(S \cup T)+v(S \cap T) \geq v(S)+v(T)$ for all $S, T \subseteq N$.

A (point-valued) solution $\psi$ on a subclass $\mathcal{C} \subseteq \mathcal{G}$ assigns a payoff vector $\psi(N, v) \in$ $\mathbb{R}^{|N|}$ to every TU-game $(N, v) \in \mathcal{C}$. Examples of solutions are the CIS-value, the ENSCvalue and the egalitarian solution. The CIS-value (see Driessen and Funaki (1991)) assigns to every player its individual worth, and distributes the remainder of the worth of the grand coalition $N$ equally among all players, i.e.,

$$
C I S_{i}(N, v)=v(\{i\})+\frac{1}{|N|}\left(v(N)-\sum_{j \in N} v(\{j\})\right) \text { for all } i \in N .
$$

The dual game $\left(N, v^{*}\right)$ of game $(N, v)$ is the game that assigns to each coalition $S \subseteq N$ the worth that is lost by the grand coalition $N$ if coalition $S$ leaves $N$, i.e.,

$$
v^{*}(S)=v(N)-v(N \backslash S) \text { for all } S \subseteq N
$$


The ENSC-value assigns to every game $(N, v)$ the CIS-value of its dual game, i.e.,

$$
\begin{aligned}
\operatorname{ENSC}_{i}(N, v) & =C I S_{i}\left(N, v^{*}\right)=v^{*}(\{i\})+\frac{1}{|N|}\left(v^{*}(N)-\sum_{j \in N} v^{*}(\{j\})\right) \\
& =v(N)-v(N \backslash\{i\})+\frac{1}{|N|}\left(v(N)-\sum_{j \in N}(v(N)-v(N \backslash\{j\}))\right) \\
& =-v(N \backslash\{i\})+\frac{1}{|N|}\left(v(N)+\sum_{j \in N} v(N \backslash\{j\})\right) \text { for all } i \in N .
\end{aligned}
$$

Thus, the ENSC-value assigns to every player in a game its marginal contribution to the 'grand coalition' and distributes the (positive or negative) remainder equally among the players. Using these two solutions we can define a class of solutions by taking any convex combination of the two, i.e., for $b \in[0,1]$ we define

$$
C I S^{b}(N, v)=b C I S(N, v)+(1-b) \operatorname{ENSC}(N, v) .
$$

A solution $\psi$ on $\mathcal{C} \subseteq \mathcal{G}$ is self-dual if $\psi(N, v)=\psi\left(N, v^{*}\right)$ for all $(N, v) \in \mathcal{C}$. The only self-dual solution in the class of solutions described above is the 'average' of the CIS and ENSC value ${ }^{2}$ obtained by taking $b=\frac{1}{2}$.

The solutions discussed above have some egalitarian flavour, in the sense that they equally split a surplus that is left after all players receive some individual payoff. Ignoring these individual payoffs we obtain the egalitarian solution given by

$$
E G A L_{i}(N, v)=\frac{1}{|N|} v(N) \text { for all } i \in N .
$$

Note that this solution is also self-dual. In this paper we discuss a class of solutions that contains all solutions $C I S^{b}, b \in[0,1]$, and the egalitarian solution.

We conclude this section by stating some well-known properties of solutions for TUgames. We call players $i, j \in N$ symmetric in game $(N, v)$ if $v(S \cup\{i\})=v(S \cup\{j\})$ for all $S \subseteq N \backslash\{i, j\}$. We call player $i \in N$ a null player in game $(N, v)$ if $v(S \cup\{i\})=v(S)$ for all $S \subseteq N \backslash\{i\}$. For game $(N, v) \in \mathcal{G}$ and permutation $\pi: N \rightarrow N$ we define the permuted game $(N, \pi v)$ by $\pi v(S)=v\left(\cup_{i \in S}\{\pi(i)\}\right)$ for all $S \subseteq N$. Finally, for $(N, v),(N, w) \in \mathcal{G}$ and $a, b \in \mathbb{R}$ we define $(N, a v+b w) \in \mathcal{G}$ by $(a v+b w)(S)=a v(S)+b w(S)$ for all $S \subseteq N$. Solution $\psi$

- is efficient on $\mathcal{C} \subseteq \mathcal{G}$ if $\sum_{i \in N} \psi_{i}(N, v)=v(N)$ for all $(N, v) \in \mathcal{C}$.

- is symmetric on $\mathcal{C} \subseteq \mathcal{G}$ if $\psi_{i}(N, v)=\psi_{j}(N, v)$ whenever $i$ and $j$ are symmetric players in $(N, v) \in \mathcal{C}$.

\footnotetext{
${ }^{2}$ Another well-known self dual solution for TU-games is the Shapley value (Shapley (1953)).
} 
- is anonymous on $\mathcal{C} \subseteq \mathcal{G}$ if for every permutation $\pi: N \rightarrow N$ it holds that $\psi_{i}(N, v)=$ $\psi_{\pi(i)}(N, \pi v)$ for every $(N, v) \in \mathcal{C}$ with $(N, \pi v) \in \mathcal{C}$.

- is linear on $\mathcal{C} \subseteq \mathcal{G}$ if $\psi(N, a v+b w)=a \psi(N, v)+b \psi(N, w)$ for all $(N, v),(N, w) \in \mathcal{C}$ and $a, b \in \mathbb{R}$ with $(N, a v+b w) \in \mathcal{C}$.

- satisfies the null player property on $\mathcal{C} \subseteq \mathcal{G}$ if $\psi_{i}(N, v)=0$ whenever $i$ is a null player in $(N, v) \in \mathcal{C}$.

- satisfies individual rationality on $\mathcal{C} \subseteq \mathcal{G}$ if $\psi_{i}(N, v) \geq v(\{i\})$ for all $i \in N$ and $(N, v) \in \mathcal{C}$.

- is non-negative on $\mathcal{C} \subseteq \mathcal{G}$ if $\psi_{i}(N, v) \geq 0$ for all $i \in N$ and $(N, v) \in \mathcal{C}$.

- reacts positive on a change in individual worth on $\mathcal{C} \subseteq \mathcal{G}$ if $\psi_{i}(N, w)>\psi_{i}(N, v)$ for every $(N, v),(N, w) \in \mathcal{C}$ with $w(\{i\})>v(\{i\})$ and $w(S)=v(S)$ for all $S \neq\{i\}$.

The first seven properties are well-known. The last property is less familiar.

\section{Two-player games}

We start by defining a class of solutions for two-player games. An $n$-player TU-game is described by $2^{n}-1$ worths of non-empty coalitions. However, in many applications it will not always be possible to know all worths or not all worths are relevant when we want to allocate the worth of the grand coalition $N$ over all players. However, in two-player games their are only three worths of non-empty coalitions, the two singletons and the grand coalition, and in these games it seems without doubt that all three worths are relevant in determining a payoff distribution. If we assume the payoff of players in a two-player game to depend linear on the worths of all coalitions we obtain solutions $\psi$ for which there exist $\alpha_{i}, \beta_{i}, \gamma_{i}, \delta_{i} \in \mathbb{R}$, such that for every two-player game $(N, v)$ with $N=\{i, j\}$, we have

$$
\psi_{i}(N, v)=\alpha_{i} v(\{i\})+\beta_{i} v(\{j\})+\gamma_{i} v(N)+\delta_{i}
$$

From Weber (1988, Theorem 1) this means that we consider linear solutions for two-player games. Next we require some well-known properties for two-player games to obtain a subclass of solutions satisfying (3.1). First we require the solution to be symmetric.

Proposition 3.1 A solution $\psi$ as given by (3.1) is symmetric if and only if $\alpha_{i}+\beta_{i}=$ $\alpha_{j}+\beta_{j}, \gamma_{i}=\gamma_{j}$ and $\delta_{i}=\delta_{j}$. 


\section{ProOF}

The if part is straightforward. To prove the only if part, suppose that $i, j \in N$ are symmetric players in $(N, v)$. By symmetry of $\psi$ it then must hold that $\psi_{i}(N, v)=\psi_{j}(N, v)$, which is equivalent to $\alpha_{i} v(\{i\})+\beta_{i} v(\{j\})+\gamma_{i} v(N)+\delta_{i}=\alpha_{j} v(\{j\})+\beta_{j} v(\{i\})+\gamma_{j} v(N)+\delta_{j}$. By symmetry of $i, j \in N$ in game $(N, v)$ we have $v(\{i\})=v(\{j\})$, and thus $\left(\alpha_{i}+\beta_{i}\right) v(\{i\})+$ $\gamma_{i} v(N)+\delta_{i}=\left(\alpha_{j}+\beta_{j}\right) v(\{i\})+\gamma_{j} v(N)+\delta_{j}$. The statement then follows since this must hold for all $(N, v) \in \mathcal{G}$ with $v(\{i\})=v(\{j\})$.

Symmetry is not sufficient to have all parameters of both players to be equal. For that we need the stronger anonymity property.

Proposition $3.2 A$ solution $\psi$ as given by (3.1) is anonymous if and only if $\alpha_{i}=\alpha_{j}, \beta_{i}=$ $\beta_{j}, \gamma_{i}=\gamma_{j}$ and $\delta_{i}=\delta_{j}$.

\section{ProOF}

Again, we just prove the only if part. Consider the permutation $\pi: N \rightarrow N$ given by $\pi(i)=j$ and $\pi(j)=i$. Then $\pi v(\{i\})=v(\{j\}), \pi v(\{j\})=v(\{i\})$ and $\pi v(N)=v(N)$. By anonymity of $\psi$ it then must hold that $\psi_{i}(N, v)=\psi_{\pi(i)}(N, \pi v)=\psi_{j}(N, \pi v)$, which is equivalent to $\alpha_{i} v(\{i\})+\beta_{i} v(\{j\})+\gamma_{i} v(N)+\delta_{i}=\alpha_{j} \pi v(\{j\})+\beta_{j} \pi v(\{i\})+\gamma_{j} \pi v(N)+\delta_{j}=$ $\alpha_{j} v(\{i\})+\beta_{j} v(\{j\})+\gamma_{j} v(N)+\delta_{j}$. The statement then follows since this must hold for all $(N, v) \in \mathcal{G}$.

If we restrict ourselves to efficient solutions then the possibilities for the values of the parameters is even further restricted.

Proposition 3.3 A solution $\psi$ as given by (3.1) is efficient if and only if $\alpha_{i}=-\beta_{j}, \alpha_{j}=$ $-\beta_{i}, \gamma_{i}+\gamma_{j}=1$ and $\delta_{i}+\delta_{j}=0$.

\section{PROOF}

Again, we just prove the only if part. Efficiency of $\psi$ implies that $\psi_{i}(N, v)+\psi_{j}(N, v)=$ $\alpha_{i} v(\{i\})+\beta_{i} v(\{j\})+\gamma_{i} v(N)+\delta_{i}+\alpha_{j} v(\{j\})+\beta_{j} v(\{i\})+\gamma_{j} v(N)+\delta_{j}=\left(\alpha_{i}+\beta_{j}\right) v(\{i\})+$ $\left(\alpha_{j}+\beta_{i}\right) v(\{j\})+\left(\gamma_{i}+\gamma_{j}\right) v(N)+\delta_{i}+\delta_{j}=v(N)$. Since this must hold for all $(N, v) \in \mathcal{G}$ we have $\alpha_{i}+\beta_{j}=\alpha_{j}+\beta_{i}=0, \gamma_{i}+\gamma_{j}=1$ and $\delta_{i}+\delta_{j}=0$.

Combining symmetry or anonymity with efficiency yields the following corollary.

Corollary 3.4 A solution $\psi$ as given by (3.1) is efficient and symmetric (or anonymous) if and only if $\alpha_{i}=\alpha_{j}=-\beta_{i}=-\beta_{j}, \gamma_{i}=\gamma_{j}=\frac{1}{2}$ and $\delta_{i}=\delta_{j}=0$. 
Proof

The statement with anonymity is immediate from Propositions 3.2 and 3.3. So, we just show that $\psi$ being efficient and symmetric implies the conditions for the parameters. $\psi$ being symmetric implies with Proposition 3.1 that $\alpha_{i}+\beta_{i}=\alpha_{j}+\beta_{j}$. $\psi$ being efficient then implies with Proposition 3.3 that $\alpha_{i}-\alpha_{j}=\alpha_{j}-\alpha_{i}$, which is equivalent to $\alpha_{i}=\alpha_{j}$. Then also $\beta_{i}=\beta_{j}$. Finally, with Proposition 3.3 it also follows that $\alpha_{i}=\alpha_{j}=-\beta_{i}=-\beta_{j}$. The conditions for $\gamma_{i}$ and $\delta_{i}, i \in N$, follow straightforward.

Note that equality of $\alpha_{i}$ and $\alpha_{j}$ is not implied by symmetry, but together with efficiency it is. (The same can be said for $\beta_{i}=\beta_{j}$ ). So, under symmetry and efficiency we can write solutions given by (3.1) as done in the following definition. For notational convenience in later sections we replace $\alpha$ by $\frac{\alpha}{2}$.

Definition 3.5 Let $\alpha \in \mathbb{R}$. A solution $\psi$ satisfies $\alpha$-standardness for two-player games if for every $(N, v) \in \mathcal{G}$ with $N=\{i, j\}, i \neq j$, it holds that

$$
\psi_{i}(N, v)=\frac{\alpha}{2}(v(\{i\})-v(\{j\}))+\frac{1}{2} v(N) \text { for } N=\{i, j\} .
$$

A solution $\psi$ satisfies weak standardness for two-player games if there exists an $\alpha \in \mathbb{R}$ such that for every $(N, v) \in \mathcal{G}$ with $|N|=2, \psi$ satisfies $\alpha$-standardness for twoplayer games.

Specific choices of $\alpha$ give different versions of standardness for two-player games as encountered in the literature:

1. Taking $\alpha=1$ yields standardness for two-player games as considered in, e.g. Hart and Mas-Colell $(1988,1989): \psi_{i}(N, v)=\frac{1}{2} v(\{i\})-\frac{1}{2} v(\{j\})+\frac{1}{2} v(N)=v(\{i\})+\frac{1}{2}(v(N)-$ $v(\{i\})-v(\{j\}))$ with $N=\{i, j\}$.

2. Taking $\alpha=0$, yields egalitarian standardness for two-player games: $\psi_{i}(N, v)=$ $\frac{1}{2} v(N)$ for $i \in N$.

In this section we showed how for two-player games the assumption that player's payoffs depend linear on the worths of all coalitions, together with symmetry and efficiency yields a weak standardness for two-player games that generalizes the regular standardness and the egalitarian standardness for two-player games. Although we will not require the null player property for our solutions, we can say that requiring efficiency, anonymity and the null player property on two-player games is equivalent to standardness for two-player games. In the next section we extend the class of solutions obtained in this section to $n$-player games using a reduced game consistency. 


\section{An extension to $n$-player games using a reduced game consistency}

Next we extend the definition of the class of solutions for two-player games satisfying weak standardness to $n$-player games using a reduced game consistency. Take a game $(N, v)$, a payoff vector $x \in \mathbb{R}^{n}$, and a player $j \in N$. The player set of a reduced game is obtained by removing player $j$ from the original player set $N$. The worths of the coalitions in this reduced game reflect what these coalitions can earn if player $j$ has left the game with its payoff $x_{j}$. The worth of the coalition $N \backslash\{j\}$ (the 'grand coalition') in the reduced game is equal to the worth of $N$ minus the payoff $x_{j}$ assigned to player $j$. Clearly, this is what is left to be allocated to the players in $N \backslash\{j\}$ after sending player $j$ out of the game with payoff $x_{j}$. For the other coalitions $S \subset N \backslash\{j\}$ we assume that every majority coalition has (or thinks it has) the cooperation of $j$ (but has to pay $x_{j}$ to $j$ ) and every minority coalition has not, i.e. coalition $S \subset N \backslash\{j\}$ earns $v(S \cup\{j\})-x_{j}$ if $|S|>\frac{|N|-1}{2}$, and it earns $v(S)$ if $|S|<\frac{|N|-1}{2}$. If $S$ is neither a majority nor a minority coalition then we assume that with probability $\beta \in[0,1]$ it does not have player $j$ 's cooperation (and thus earns $v(S)$ ) and with probability $1-\beta$ it has the cooperation of $j$ (and earns $v(S \cup\{j\})-x_{j}$ ). This yields the following reduced game.

Definition 4.1 Take $\beta \in[0,1]$. Given a game $(N, v) \in \mathcal{C}$ with $|N| \geq 3$, a player $j \in N$, and a payoff vector $x \in \mathbb{R}^{N}$, the $\beta$-reduced game with respect to $j$ and $x$ is the game $\left(N \backslash\{j\}, v^{x, \beta}\right)$ given by

$$
v^{x, \beta}(S)= \begin{cases}v(N)-x_{j} & \text { if } S=N \backslash\{j\} \\ v(S \cup\{j\})-x_{j} & \text { if } S \subset N \backslash\{j\} \text { with } \frac{|N|-1}{2}<|S|<|N|-1 \\ \beta v(S)+(1-\beta)\left(v(S \cup\{j\})-x_{j}\right) & \text { if } S \subset N \backslash\{j\} \text { with }|S|=\frac{|N|-1}{2} \\ v(S) & \text { if } S \subset N \backslash\{j\} \text { with }|S|<\frac{|N|-1}{2} .\end{cases}
$$

Note that the case $|S|=\frac{|N|-1}{2}$ does not occur in case $|N|$ is even. The characterization result we present below holds if we take a qualified majority $q \in\{2, \ldots,|N|-3\}$ instead of the simple majority rule. Anyway we require that there exist both majority and minority coalitions, and thus we do not allow the quota $q$ to be equal to 1 or $|N|-2$. Taking a qualified majority with $q=1$ we would obtain a reduced game that is similar to the reduced games considered in Funaki and Yamato (2001).

We are ready to give a definition of the consistency property of a solution associated with a reduced game.

Definition 4.2 Let $\psi$ be a solution on $\mathcal{C} \subseteq \mathcal{G}$, and $\beta \in[0,1]$. Solution $\psi$ satisfies $\beta$ consistency on $\mathcal{C}$ if and only if for every $(N, v) \in \mathcal{C}$ with $|N| \geq 3, j \in N$, and $x=\psi(N, v)$ it holds that $\left(N \backslash\{j\}, v^{x, \beta}\right) \in \mathcal{C}$ and $\psi_{i}\left(N \backslash\{j\}, v^{x, \beta}\right)=\psi_{i}(N, v)$ for $i \in N \backslash\{j\}$. 
Consistency implies that given a game $(N, v)$, if $x$ is a solution payoff vector for $(N, v)$, then for every player $j \in N$, the payoff vector $x_{N \backslash\{j\}}$ with payoffs for the players in $N \backslash\{j\}$, must be a solution payoff vector of the reduced game $\left(N \backslash\{j\}, v^{x, \beta}\right)$. It is a kind of internal consistency requirement to guarantee that players respect the recommendations made by the solution. With a slight abuse of notation we denote in the remainder of the paper the characteristic function $v^{x, \beta}$ just by $v^{x}$.

Next we show that for $\alpha \in \mathbb{R}$ and $\beta \in[0,1], \alpha$-standardness for two-player games and $\beta$-consistency implies that the solution is uniquely determined and is given by

$$
\varphi_{i}^{\alpha, \beta}(N, v)=\lambda_{i}^{\alpha, \beta}(N, v)+\frac{1}{|N|}\left(v(N)-\sum_{j \in N} \lambda_{j}^{\alpha, \beta}(N, v)\right),
$$

where $\lambda_{i}^{\alpha, \beta}(N, v)=\alpha(\beta v(\{i\})-(1-\beta) v(N \backslash\{i\}))$ for $i \in N$. We denote the class of all solutions that are obtained in this way by $\Phi:=\left\{\varphi^{\alpha, \beta} \mid \alpha \in \mathbb{R}, \beta \in[0,1]\right\}$. These all have some egalitarian flavour. They give each player $i$ in a game $(N, v)$ the value $\lambda_{i}^{\alpha, \beta}(N, v)$, and the remainder of $v(N)$ is equally split among all players. Since for two-player games $(N, v)$ with $N=\{i, j\}$ it holds that $v(\{i\})=v(N \backslash\{j\})$, the payoff vector assigned to twoplayer games does not depend on $\beta$. Obviously, applied to two-player games the solution $\varphi^{\alpha, \beta}$ satisfies $\alpha$-standardness for two player games. Next we show that it also satisfies $\beta$-consistency.

Proposition 4.3 Take any $\beta \in[0,1]$. For every $\alpha \in \mathbb{R}$ the solution $\varphi^{\alpha, \beta}$ satisfies $\beta$ consistency on the class of all games $\mathcal{G}$.

\section{PROOF}

Take any $\beta \in[0,1]$ and fix $\alpha \in \mathbb{R}$. Take any $(N, v) \in \mathcal{G}$. First, suppose that $|N| \geq 4$. For $x=\varphi^{\alpha, \beta}(N, v)$ we have

$$
\begin{aligned}
\lambda_{i}^{\alpha, \beta}(N \backslash\{j\}, & \left.v^{x}\right)=\alpha\left(\beta v^{x}(\{i\})-(1-\beta) v^{x}(N \backslash\{i, j\})\right) \\
& =\alpha\left(\beta v(\{i\})-(1-\beta)\left(v(N \backslash\{i\})-x_{j}\right)\right)=\lambda_{i}^{\alpha, \beta}(N, v)+(1-\beta) x_{j}
\end{aligned}
$$

since in that case $|\{i\}|<\frac{|N|-1}{2}$ and $|N \backslash\{i, j\}|>\frac{|N|-1}{2}$. Put $\lambda_{i}=\lambda_{i}^{\alpha, \beta}(N, v)$ and $\lambda_{i}^{x}=$ $\lambda_{i}^{\alpha, \beta}\left(N \backslash\{j\}, v^{x}\right)$ with $x=\varphi^{\alpha, \beta}(N, v), i \in N$. Then with (4.3) it follows that

$$
\begin{aligned}
& \varphi_{i}^{\alpha, \beta}\left(N \backslash\{j\}, v^{x}\right)=\lambda_{i}^{x}+\frac{1}{|N|-1}\left(v^{x}(N \backslash\{j\})-\sum_{k \in N \backslash\{j\}} \lambda_{k}^{x}\right) \\
& =\lambda_{i}+(1-\beta) x_{j}+\frac{1}{|N|-1}\left(v(N)-x_{j}-\sum_{k \in N \backslash\{j\}}\left(\lambda_{k}+(1-\beta) x_{j}\right)\right)
\end{aligned}
$$




$$
\begin{aligned}
& =\lambda_{i}+(1-\beta) x_{j}+\frac{1}{|N|-1}\left(v(N)-\lambda_{j}-\frac{1}{|N|}\left(v(N)-\sum_{k \in N} \lambda_{k}\right)-\sum_{k \in N \backslash\{j\}} \lambda_{k}\right)-(1-\beta) x_{j} \\
& =\lambda_{i}+\frac{1}{|N|-1}\left(\frac{|N|-1}{|N|}\left(v(N)-\sum_{k \in N} \lambda_{k}\right)\right)=\varphi_{i}^{\alpha, \beta}(N, v) .
\end{aligned}
$$

Second, consider the case $|N|=3$. Let $x=\varphi^{\alpha, \beta}(N, v), \bar{\beta}=1-\beta$ and $N=\{i, j, k\}$. (Note that $|N|=3$ implies that $i, j$ and $k$ are distinct players.) First we remark that

$$
\begin{aligned}
& \varphi_{i}^{\alpha, \beta}(N, v)=\alpha(\beta v(\{i\})-\bar{\beta} v(N \backslash\{i\}))+\frac{1}{3}\left(v(N)-\sum_{l \in N} \alpha(\beta v(\{l\})-\bar{\beta} v(N \backslash\{l\}))\right) \\
& =\alpha(\beta v(\{i\})-\bar{\beta} v(\{j, k\})) \\
& +\frac{1}{3}(v(N)-\alpha \beta v(\{i\})-\alpha \beta v(\{j\})-\alpha \beta v(\{k\})+\alpha \bar{\beta} v(\{j, k\})+\alpha \bar{\beta} v(\{k, i\})+\alpha \bar{\beta} v(\{i, j\})) .
\end{aligned}
$$

From the definitions of the solution and the reduced game, and the fact that for this three player game $|\{i\}|=|\{j\}|=\frac{|N|-1}{2}$ we have for $x=\varphi^{\alpha, \beta}(N, v)$

$$
\begin{aligned}
& \varphi_{i}^{\alpha, \beta}\left(N \backslash\{k\}, v^{x}\right)=\varphi_{i}^{\alpha, \beta}\left(\{i, j\}, v^{x}\right)=\frac{\alpha}{2}\left(v^{x}(\{i\})-v^{x}(\{j\})\right)+\frac{1}{2} v^{x}(\{i, j\}) \\
& =\frac{\alpha}{2}\left(\left(\beta v(\{i\})+\bar{\beta} v(\{i, k\})-\bar{\beta} x_{k}\right)-\left(\beta v(\{j\})+\bar{\beta} v(\{j, k\})-\bar{\beta} x_{k}\right)\right) \\
& \quad+\frac{1}{2}[v(N)-\alpha(\beta v(\{k\})-\bar{\beta} v(\{i, j\})) \\
& \left.\quad-\frac{1}{3}(v(N)-\alpha \beta v(\{i\})-\alpha \beta v(\{j\})-\alpha \beta v(\{k\})+\alpha \bar{\beta} v(\{j, k\})+\alpha \bar{\beta} v(\{k, i\})+\alpha \bar{\beta} v(\{i, j\}))\right] \\
& =(\alpha \beta v(\{i\})-\alpha \bar{\beta} v(\{j, k\}))+\frac{1}{2}(-\alpha \beta v(\{i\})+\alpha \bar{\beta} v(\{i, k\})-\alpha \beta v(\{j\})+\alpha \bar{\beta} v(\{j, k\})) \\
& \left.\quad+\frac{1}{3}(v(N)-\alpha \beta v(\{k\})+\alpha \bar{\beta} v(\{i, j\}))\right)-\frac{1}{6}(-\alpha \beta v(\{i\})-\alpha \beta v(\{j\})+\alpha \bar{\beta} v(\{j, k\})+\alpha \bar{\beta} v(\{k, i\})) \\
& =\alpha(\beta v(\{i\})-\bar{\beta} v(\{j, k\})) \\
& \left.\quad+\frac{1}{3}(v(N)-\alpha \beta v(\{k\})+\alpha \bar{\beta} v(\{i, j\}))-\alpha \beta v(\{i\})-\alpha \beta v(\{j\})+\alpha \bar{\beta} v(\{j, k\})+\alpha \bar{\beta} v(\{k, i\})\right) \\
& =\varphi_{i}^{\alpha, \beta}(N, v) .
\end{aligned}
$$

This completes the proof.

Next we characterize the solutions $\varphi^{\alpha, \beta}$.

Theorem 4.4 Take any $\beta \in[0,1]$ and $\alpha \in \mathbb{R}$. A solution $\psi$ satisfies efficiency, $\beta$ consistency and $\alpha$-standardness for two-player games on the class of all games $\mathcal{G}$, if and only if $\psi=\varphi^{\alpha, \beta}$. 


\section{Proof}

$\varphi^{\alpha, \beta}$ satisfying efficiency and $\alpha$-standardness for two-player games is straightforward. $\varphi^{\alpha, \beta}$ satisfying $\beta$-consistency follows from Propositon 4.3. Here we prove the only if part. Take $\beta \in[0,1]$ and $\alpha \in \mathbb{R}$, and let $\psi$ be a solution which satisfies efficiency, $\beta$-consistency and $\alpha$-standardness for two-player games. If $|N|=2$ then $\psi(N, v)=\varphi^{\alpha, \beta}$ follows from $\alpha$-standardness for two-player games.

Consider a game $(N, v) \in \mathcal{G}$ with $|N| \geq 3$, and let $x=\psi(N, v)$. Fix $i, j \in N(i \neq j)$. Let $N=\left\{i, j, k_{1}, k_{2}, \ldots k_{n-2}\right\}$. By using the $\beta$-consistency of $\psi$ repeatedly, we have

$$
\begin{aligned}
& \psi_{i}(N, v)-\psi_{j}(N, v)=\psi_{i}\left(N \backslash\left\{k_{n-2}\right\}, v^{x}\right)-\psi_{j}\left(N \backslash\left\{k_{n-2}\right\}, v^{x}\right) \\
& =\psi_{i}\left(N \backslash\left\{k_{n-2}, k_{n-3}\right\},\left(v^{x}\right)^{x}\right)-\psi_{j}\left(N \backslash\left\{k_{n-2}, k_{n-3}\right\},\left(v^{x}\right)^{x}\right) \\
& =\psi_{i}\left(N \backslash\left\{k_{n-2}, k_{n-3}, \ldots, k_{1}\right\},\left(\ldots\left(\left(v^{x}\right)^{x}\right) \ldots\right)^{x}\right)-\psi_{j}\left(N \backslash\left\{k_{n-2}, k_{n-3}, \ldots, k_{1}\right\},\left(\ldots\left(\left(v^{x}\right)^{x}\right) \ldots\right)^{x}\right) \\
& =\psi_{i}\left(\{i, j\},\left(\ldots\left(\left(v^{x}\right)^{x}\right) \ldots\right)^{x}\right)-\psi_{j}\left(\{i, j\},\left(\ldots\left(\left(v^{x}\right)^{x}\right) \ldots\right)^{x}\right) .
\end{aligned}
$$

The $\alpha$-standardness for two-player games implies that

$$
\begin{aligned}
& \psi_{i}(N, v)-\psi_{j}(N, v) \\
& \left.\left.\left.\left.=\frac{\alpha}{2}\left(\left(\ldots\left(\left(v^{x}\right)^{x}\right) \ldots\right)^{x}\right)(\{i\})-\left(\ldots\left(\left(v^{x}\right)^{x}\right) \ldots\right)^{x}\right)(\{j\})\right)-\frac{\alpha}{2}\left(\left(\ldots\left(\left(v^{x}\right)^{x}\right) \ldots\right)^{x}\right)(\{j\})-\left(\ldots\left(\left(v^{x}\right)^{x}\right) \ldots\right)^{x}\right)(\{i\})\right) \\
& \left.\left.=\alpha\left(\left(\ldots\left(\left(v^{x}\right)^{x}\right) \ldots\right)^{x}\right)(\{i\})-\left(\ldots\left(\left(v^{x}\right)^{x}\right) \ldots\right)^{x}\right)(\{j\})\right)
\end{aligned}
$$

The definition of the reduced game implies that

$$
\begin{aligned}
& \left(\left(\ldots\left(\left(v^{x}\right)^{x}\right) \ldots\right)^{x}\right)(\{i\})=\beta v(\{i\})+(1-\beta) v\left(\left\{i, k_{1}\right\}\right)-(1-\beta) x_{k_{1}} \\
& \left(\left(\ldots\left(\left(v^{x}\right)^{x}\right) \ldots\right)^{x}\right)(\{j\})=\beta v(\{j\})+(1-\beta) v\left(\left\{j, k_{1}\right\}\right)-(1-\beta) x_{k_{1}} .
\end{aligned}
$$

Here each last equation is obtained from the definition of the reduced game of three-player games. Then we have

$$
\psi_{i}(N, v)-\psi_{j}(N, v)=\alpha \beta(v(\{i\})-v(\{j\}))+\alpha(1-\beta)\left(v\left(\left\{i, k_{1}\right\}\right)-v\left(\left\{j, k_{1}\right\}\right)\right) .
$$

On the other hand, since the solution $\varphi^{\alpha, \beta}$ also satisfies $\beta$-consistency, we have

$$
\varphi_{i}^{\alpha, \beta}(N, v)-\varphi_{j}^{\alpha, \beta}(N, v)=\alpha \beta(v(\{i\})-v(\{j\}))+\alpha(1-\beta)\left(v\left(\left\{i, k_{1}\right\}\right)-v\left(\left\{j, k_{1}\right\}\right)\right),
$$

by the same argument. This implies

$$
\psi_{i}(N, v)-\psi_{j}(N, v)=\varphi_{i}^{\alpha, \beta}(N, v)-\varphi_{j}^{\alpha, \beta}(N, v)
$$

for any pair $i, j \in N,(i \neq j)$. 
These equations together with efficiency imply

$$
\psi_{i}(N, v)=\varphi_{i}^{\alpha, \beta}(N, v)
$$

for all $i \in N$. This completes the proof.

We remark that for the characterization given above it is not necessary to have the parameter $\beta$ enter the reduced game for more than three player games. If $|N| \geq 4$, instead of $\beta$ we could take any fixed $b \in[0,1]$ to obtain a characterization. However, it is crucial that the parameter $\beta$ appears in the reduced game for three player games ${ }^{3}$.

In the previous section we said that a solution satisfies weak standardness for twoplayer games if and only if there exists an $\alpha \in \mathbb{R}$ such that it satisfies $\alpha$-standardness for two-player games. Similar we can say that a solution satisfies weak consistency if and only if there exists a $\beta \in[0,1]$ such that it satisfies $\beta$-consistency. In a similar way as the proof of Theorem 4.4 it can be shown that these two weaker properties with efficiency characterize the class of solutions $\Phi$.

Corollary 4.5 A solution $\psi$ satisfies efficiency, weak consistency and weak standardness for two-player games on the class of all games $\mathcal{G}$, if and only if $\psi \in \Phi$.

The $\alpha$-standardness for two-player games and $\beta$-consistency also make clear how the solutions $\varphi^{\alpha, \beta}$ depend on these two parameters. The parameter $\alpha \in \mathbb{R}$ determines the payoff distribution in two-player games, while the parameter $\beta \in[0,1]$ determines the probability about cooperation of the leaving player $j$ in the coalitions in the reduced game that are neither majority nor minority coalitions in those games.

We end this section by stating that adding a constant times $v(N)$ to $\lambda^{\alpha, \beta}$ yields the same solution.

Proposition 4.6 For every $(N, v) \in \mathcal{G}$ it holds that

$$
\varphi_{i}^{\alpha, \beta}(N, v)=\bar{\lambda}_{i}(N, v)+\frac{1}{|N|}\left(v(N)-\sum_{j \in N} \bar{\lambda}_{j}(N, v)\right)
$$

if $\bar{\lambda}(N, v)=\lambda^{\alpha, \beta}(N, v)+k v(N), k>0$.

Proof

For $(N, v) \in \mathcal{G}$ and $k>0$ it holds that $\bar{\lambda}_{i}(N, v)+\frac{1}{|N|}\left(v(N)-\sum_{j \in N} \bar{\lambda}_{j}(N, v)\right)$

$=\lambda_{i}^{\alpha, \beta}(N, v)+k v(N)+\frac{1}{|N|}\left(v(N)-\sum_{j \in N} \lambda_{j}^{\alpha, \beta}(N, v)-\sum_{j \in N} k v(N)\right)=\lambda_{i}^{\alpha, \beta}(N, v)+k v(N)+$ $\frac{1}{|N|}\left(v(N)-\sum_{j \in N} \lambda_{j}^{\alpha, \beta}(N, v)\right)-\frac{|N|}{|N|} k v(N)=\varphi_{i}^{\alpha, \beta}(N, v)$.

\footnotetext{
${ }^{3}$ We give an illustration in the appendix.
} 


\section{$5 \quad$ Some specific solutions}

In this section we discuss some specific solutions in the class $\Phi$ defined in the previous sections. Some specific solutions in $\Phi$ are the following:

1. The CIS-value is obtained by taking $\alpha=\beta=1$, i.e., $C I S(N, v)=\varphi^{1,1}(N, v)$.

2. Taking $\beta=1, \alpha \in[0,1]$ we obtain a solution that assigns to every player a fraction $\alpha$ of its individual worth, and distributes the remainder of $v(N)$ equally among all players.

3. The ENSC-value is obtained by taking $\alpha=1, \beta=0$, i.e., $\operatorname{ENSC}(N, v)=\varphi^{1,0}(N, v)$.

4. By taking $\alpha=1, \beta=b$ we obtain any convex combination $C I S^{b}$ of the CIS-value and ENSC-value, i.e., $C I S^{b}(N, v)=\varphi^{1, b}(N, v)$.

5. Taking $\alpha=0$ we obtain the egalitarian solution, i.e., $\operatorname{EGAL}(N, v)=\varphi^{0, \beta}(N, v), \beta \in$ $[0,1]$

6. Besides taking convex combinations of the CIS- and ENSC-value, also any convex combination of the CIS-value and the egalitarian solution belongs to $\Phi$. More general, any convex combination of $C I S^{b}$ (i.e. any convex combination of the CIS- and ENSC value) and the egalitarian solution can be obtained:

$\varphi_{i}^{\alpha, \beta}(N, v)$

$=\alpha(\beta v(\{i\})-(1-\beta) v(N \backslash\{i\})))+\frac{1}{|N|}\left(v(N)-\sum_{j \in N} \alpha(\beta v(\{j\})-(1-\beta) v(N \backslash\{j\}))\right)$

$=\alpha(\beta v(\{i\})-(1-\beta) v(N \backslash\{i\})))+\frac{\alpha}{|N|}\left(v(N)-\sum_{j \in N}(\beta v(\{j\})-(1-\beta) v(N \backslash\{j\}))+\frac{1-\alpha}{|N|} v(N)\right.$

$=\alpha C I S^{\beta}(N, v)+(1-\alpha) E G A L(N, v)$

for $\alpha \in \mathbb{R}, \beta \in[0,1]$.

\subsection{Some relations between solutions in $\Phi$}

From the relations between convex combinations of the CIS- and ENSC-value (see Examples 1,3 , and 4 above) we find the relation

$$
\varphi^{1, \beta}(N, v)=\beta \varphi^{1,1}(N, v)+(1-\beta) \varphi^{1,0}(N, v), \beta \in[0,1] .
$$


With the convex combinations of these solutions and the egalitarian solution (see Examples 5 and 6 above) we can generalize (5.4) to

$\varphi^{\alpha, \beta}(N, v)=\alpha \varphi^{1, \beta}(N, v)+(1-\alpha) \varphi^{0,1}(N, v)=\alpha \beta \varphi^{1,1}(N, v)+\alpha(1-\beta) \varphi^{1,0}(N, v)+(1-\alpha) \varphi^{0,1}(N, v)$ for $\alpha, \beta \in[0,1]$.

Next we present two more relations between solutions in $\Phi$. In the following we often use the expression

$\varphi_{i}^{\alpha, \beta}(N, v)=\alpha \beta v(\{i\})-\alpha(1-\beta) v(N \backslash\{i\})+\frac{1}{|N|} v(N)-\frac{1}{|N|} \alpha \beta \sum_{j \in N} v(\{j\})+\frac{1}{|N|} \alpha(1-\beta) \sum_{j \in N} v(N \backslash\{j\})$

Proposition 5.1 For every $\alpha \in \mathbb{R}, \beta \in[0,1]$ and $(N, v) \in \mathcal{G}$ it holds that

1. $\varphi^{\alpha, \beta}(N, v)+\varphi^{\alpha, 1-\beta}(N, v)=\varphi^{\alpha, 1}(N, v)+\varphi^{\alpha, 0}(N, v)$;

2. $\varphi^{\alpha, \beta}(N, v)+\varphi^{\gamma, \beta}(N, v)=\varphi^{\alpha+\gamma, \beta}(N, v)+\varphi^{0, \beta}(N, v)$.

\section{ProOF}

Let $\alpha \in \mathbb{R}, \beta \in[0,1]$ and $(N, v) \in \mathcal{G}$. With (5.5) it then follows that 1.

$$
\begin{aligned}
& \varphi^{\alpha, \beta}(N, v)+\varphi^{\alpha, 1-\beta}(N, v) \\
& =\alpha \beta v(\{i\})-\alpha(1-\beta) v(N \backslash\{i\})+\frac{1}{|N|} v(N)-\frac{1}{|N|} \alpha \beta \sum_{j \in N} v(\{j\}) \\
& \left.\quad+\frac{1}{|N|} \alpha(1-\beta) \sum_{j \in N} v(N \backslash\{j\})\right)+\alpha(1-\beta) v(\{i\})-\alpha(1-(1-\beta)) v(N \backslash\{i\}) \\
& \left.\quad+\frac{1}{|N|} v(N)-\frac{1}{|N|} \alpha(1-\beta) \sum_{j \in N} v(\{j\})+\frac{1}{|N|} \alpha(1-(1-\beta)) \sum_{j \in N} v(N \backslash\{j\})\right) \\
& =\alpha v(\{i\})+\frac{1}{|N|}\left(v(N)-\alpha \sum_{j \in N} v(\{j\})\right)-\alpha(v(N \backslash\{i\})) \\
& \quad+\frac{1}{|N|}\left(v(N)+\alpha \sum_{j \in N} v(N \backslash\{j\})\right) \\
& =\varphi^{\alpha, 1}(N, v)+\varphi^{\alpha, 0}(N, v),
\end{aligned}
$$

and

2 .

$$
\varphi^{\alpha, \beta}(N, v)+\varphi^{\gamma, \beta}(N, v)=
$$




$$
\begin{aligned}
=\alpha & \beta v(\{i\})-\alpha(1-\beta) v(N \backslash\{i\})+\frac{1}{|N|} v(N)-\frac{1}{|N|} \alpha \beta \sum_{j \in N} v(\{j\}) \\
& +\frac{1}{|N|} \alpha(1-\beta) \sum_{j \in N} v(N \backslash\{j\})+\gamma \beta v(\{i\})-\gamma(1-\beta) v(N \backslash\{i\})+\frac{1}{|N|} v(N) \\
& \quad-\frac{1}{|N|} \gamma \beta \sum_{j \in N} v(\{j\})+\frac{1}{|N|} \gamma(1-\beta) \sum_{j \in N} v(N \backslash\{j\}) \\
=( & +\gamma)(\beta v(\{i\})-(1-\beta) v(N \backslash\{i\})) \\
& +\frac{1}{|N|}\left(v(N)-(\alpha+\gamma) \sum_{j \in N} \beta(v(\{j\})-(1-\beta) v(N \backslash\{j\}))\right)+\frac{1}{|N|} v(N) \\
= & \varphi^{\alpha+\gamma, \beta}(N, v)+\varphi^{0, \beta}(N, v) .
\end{aligned}
$$

In the remainder of this section we give characterizations of some specific solutions in $\Phi$, in particular the CIS-value, the ENSC-value, their average and the egalitarian solution.

\subsection{Characterizations using standardness and reduced game con- sistency}

We begin by giving characterizations of some specific solutions by using specific cases of $\alpha$-standardness for two-player games and $\beta$-consistency. By taking particular values for $\beta$ in Theorem 4.4 we obtain axiomatic characterizations of specific classes $\Phi^{b}, b \in[0,1]$. Taking also particular values for $\alpha$ (which can be reflected in a more specific standardness for two-player games) gives axiomatic characterizations of specific solutions in $\Phi$. These characterizations follow directly from Theorem 4.4, and therefore are stated without further proof.

Taking $\alpha=1$ we obtain characterizations of all convex combinations $\varphi^{1, \beta}, \beta \in[0,1]$, of the CIS- and ENSC value. Note that taking $\alpha=1$ is equivalent to requiring the solution to satisfy standardness for two-player games.

Corollary 5.2 Take any $\beta \in[0,1]$. A solution $\psi$ satisfies $\beta$-consistency, standardness for two-player games and efficiency if and only if $\psi$ is the $C I S^{\beta}$ value $\varphi^{1, \beta}$.

Taking $\beta=\alpha=1$ we obtain a characterization of the CIS-value. Note that $\beta=1$ yields 1-consistency meaning consistency associated with the reduced game $\left(N \backslash\{j\}, v^{x}\right), j \in N$, $x \in \mathbb{R}^{n}$, given by 


$$
v^{x}(S)= \begin{cases}v(N)-x_{j} & \text { if } S=N \backslash\{j\} \\ v(S \cup\{j\})-x_{j} & \text { if } S \subset N \backslash\{j\} \text { with } \frac{|N|-1}{2}<|S|<|N|-1 \\ v(S) & \text { if } S \subset N \backslash\{j\} \text { with }|S| \leq \frac{|N|-1}{2} .\end{cases}
$$

So, to have the participation of the leaving player $j$ a coalition needs a strict majority in the reduced game.

Corollary 5.3 A solution $\psi$ satisfies 1-consistency, standardness for two-player games and efficiency if and only if $\psi$ is the CIS-value $\varphi^{1,1}$.

Taking $\beta=0$ and $\alpha=1$ we obtain a characterization of the ENSC-value. Note that $\beta=0$ yields 0 -consistency meaning consistency associated with the reduced game $\left(N \backslash\{j\}, v^{x}\right)$, $j \in N$ and $x \in \mathbb{R}^{n}$, given by

$$
v^{x}(S)= \begin{cases}v(N)-x_{j} & \text { if } S=N \backslash\{j\} \\ v(S \cup\{j\})-x_{j} & \text { if } S \subset N \backslash\{j\} \text { with } \frac{|N|-1}{2} \leq|S|<|N|-1 \\ v(S) & \text { if } S \subset N \backslash\{j\} \text { with }|S|<\frac{|N|-1}{2} .\end{cases}
$$

So, to have the participation of the leaving player $j$ a strict majority in the reduced game is not necessary.

Corollary 5.4 A solution $\psi$ satisfies 0-consistency, standardness for two-player games and efficiency if and only if $\psi$ is the ENSC-value $\varphi^{1,0}$.

Note that according to the above two corollaries the difference between the CIS- and ENSCvalue is only with respect to how we treat coalitions that are neither majority nor minority coalitions in the reduced game.

Besides taking $\beta=0$ or $\beta=1$ as done above we can, for example, take $\beta=\frac{1}{2}$. This yields in the reduced game that coalitions that are not majority nor minority coalitions have the leaving player's cooperation with probability $\frac{1}{2}$. The corresponding solution is the average of the CIS- and ENSC value.

Finally, we mention that taking egalitarian standardness for two player games (i.e. taking $\alpha=0$ ) yields a characterization of the egalitarian solution.

Corollary 5.5 Take any $\beta \in[0,1]$. A solution $\psi$ satisfies $\beta$-consistency, egalitarian standardness for two-player games and efficiency if and only if $\psi$ is the egalitarian solution.

Note that the last corollary implies that the egalitarian solution is axiomatized by egalitarian standardness for two-player games, efficiency and any consistency as discussed in this section. 


\subsection{Characterizations using self-duality}

In this subsection we provide characterizations of some solutions in $\Phi$ using self-duality. Although these characterizations can be stated for fixed player set $N$, we continue our previous notation and consider the class of all games $\mathcal{G}$.

Before using self-duality in characterizing some solutions in $\Phi$, we state that the class of solutions $\Phi$ itself is closed under duality, meaning that for every solution in this class it holds that its dual solution is also in the class. More specific, we state the following property.

Proposition 5.6 For every $\alpha \in \mathbb{R}, \beta \in[0,1]$ and $(N, v) \in \mathcal{G}$ it holds that $\varphi^{\alpha, \beta}\left(N, v^{*}\right)=$ $\varphi^{\alpha, 1-\beta}(N, v)$.

\section{PROOF}

Let $\alpha \in \mathbb{R}, \beta \in[0,1]$ and $(N, v) \in \mathcal{G}$. Then

$$
\begin{aligned}
& \varphi^{\alpha, \beta}\left(N, v^{*}\right)=\lambda_{i}^{\alpha, \beta}\left(N, v^{*}\right)+\frac{1}{|N|}\left(v(N)-\sum_{j \in N} \lambda_{j}^{\alpha, \beta}\left(N, v^{*}\right)\right) \\
= & \alpha\left(\beta v^{*}(\{i\})-(1-\beta) v^{*}(N \backslash\{i\})\right)+\frac{1}{|N|}\left(v(N)-\alpha \sum_{j \in N}\left(\beta v^{*}(\{j\})-(1-\beta) v^{*}(N \backslash\{j\})\right)\right) \\
= & \alpha(\beta v(N)-\beta v(N \backslash\{i\})-(1-\beta) v(N)+(1-\beta) v(\{i\})) \\
& +\frac{1}{|N|}\left(v(N)-\alpha \sum_{j \in N}(\beta v(N)-\beta v(N \backslash\{j\})-(1-\beta) v(N)+(1-\beta) v(\{j\}))\right) \\
= & v(N)\left(\alpha \beta-\alpha(1-\beta)+\frac{1}{|N|}-\frac{|N| \alpha \beta}{|N|}+\frac{|N| \alpha(1-\beta)}{|N|}\right) \\
& +\alpha\left((1-\beta) v(\{i\})-\beta v(N \backslash\{i\})+\frac{1}{|N|} \sum_{j \in N}(\beta v(N \backslash\{j\})-(1-\beta) v(\{j\}))\right) \\
= & \frac{1}{|N|} v(N)+\alpha((1-\beta) v(\{i\})-\beta v(N \backslash\{i\}))-\frac{1}{|N|}\left(\sum_{j \in N} \alpha((1-\beta) v(\{j\})-\beta v(N \backslash\{j\}))\right) \\
= & \varphi^{\alpha, 1-\beta}(N, v) .
\end{aligned}
$$

Although the class $\Phi$ is closed under duality, this does not imply that every solution in $\Phi$ is self-dual. Taking $\alpha=0$ or $\beta=\frac{1}{2}$ yields self-duality. The corresponding solutions are the only self dual solutions in the class $\Phi$. 
Proposition 5.7 Consider solution $\varphi^{\alpha, \beta} \in \Phi$. Then $\varphi^{\alpha, \beta}$ is self dual if and only if $\alpha=0$ or $\beta=\frac{1}{2}$.

\section{Proof}

From Proposition 5.6 it is straightforward to show that these solutions are self-dual. To show that these are the only self-dual solutions in the class $\Phi$, suppose that $\varphi^{\alpha, \beta}(N, v)=$ $\varphi^{\alpha, \beta}\left(N, v^{*}\right)$ for all $(N, v) \in \mathcal{G}$. Then with Proposition 5.6 we have

$$
\varphi^{\alpha, \beta}(N, v)=\varphi^{\alpha, \beta}\left(N, v^{*}\right)
$$

$\Leftrightarrow$

$$
\varphi^{\alpha, \beta}(N, v)=\varphi^{\alpha, 1-\beta}(N, v)
$$

$\Leftrightarrow$

$$
\lambda_{i}^{\alpha, \beta}(N, v)+\frac{1}{|N|}\left(v(N)-\sum_{j \in N} \lambda_{j}^{\alpha, \beta}(N, v)\right)=\lambda_{i}^{\alpha, 1-\beta}(N, v)+\frac{1}{|N|}\left(v(N)-\sum_{j \in N} \lambda_{j}^{\alpha, 1-\beta}(N, v)\right)
$$

$\Leftrightarrow$

$$
\begin{aligned}
& \alpha(\beta v(\{i\})-(1-\beta) v(N \backslash\{i\}))+\frac{1}{|N|}\left(v(N)-\sum_{j \in N} \alpha(\beta v(\{j\})-(1-\beta) v(N \backslash\{j\}))\right) \\
& =\alpha((1-\beta) v(\{i\})-\beta v(N \backslash\{i\}))+\frac{1}{|N|}\left(v(N)-\sum_{j \in N} \alpha((1-\beta) v(\{j\})-\beta v(N \backslash\{j\}))\right)
\end{aligned}
$$

Since this must hold for all $(N, v) \in \mathcal{G}$ we have $\alpha \beta=\alpha(1-\beta)$, which is equivalent to $\alpha(2 \beta-1)=0$. So, $\alpha=0$ or $\beta=\frac{1}{2}$.

So, the only self-dual solutions in $\Phi$ are the egalitarian solution, the average of the CISand ENSC-value, and all convex combinations of these two solutions. Now we can add more properties to distinguish between the egalitarian solution (with $\alpha=0$ ) and the other self dual solutions. The egalitarian solution is the only self dual solution satisfying nonnegativeness for non-negative games.

Proposition 5.8 Consider solution $\varphi^{\alpha, \beta} \in \Phi$. Then $\varphi^{\alpha, \beta}$ is self dual and non-negative for non-negative games if and only if it is the egalitarian solution.

\section{PROOF}

Obviously the egalitarian solution is self-dual and non-negative for non-negative games. To prove that it is the only solution in $\Phi$ that is self-dual and non-negative for non-negative 
games, take a game $(N, v) \in \mathcal{G}$ with $|N| \geq 3$ such that for some $i \in N, v(\{i\})=1$, and $v(S)=0$ for $S \neq\{i\}$. (This is also called the standard game of $\{i\}$.) With (5.5) it then follows that

$$
\varphi_{i}^{\alpha, \beta}(N, v)=\alpha \beta-\frac{1}{|N|} \alpha \beta=\left(1-\frac{1}{|N|}\right) \alpha \beta .
$$

Since $v(S) \geq 0$ for all $S \subseteq N$, non-negativeness of $\varphi^{\alpha, \beta}$ implies that $\left(1-\frac{1}{|N|}\right) \alpha \beta \geq 0$. So, $\alpha \beta \geq 0$ since $1>\frac{1}{|N|}$.

Next, take a game $(N, \bar{v}) \in \mathcal{G}$ such that for some $i \in N, \bar{v}(S)=1$ if $S=\{j\}, j \neq i$, and $\bar{v}(S)=0$ otherwise. With (5.5) it then follows that

$$
\varphi_{i}^{\alpha, \beta}(N, \bar{v})=-\frac{1}{|N|} \sum_{j \in N \backslash\{i\}} \alpha \beta=-\left(\frac{|N|-1}{|N|}\right) \alpha \beta .
$$

Since $\bar{v}(S) \geq 0$ for all $S \subseteq N$, non-negativeness of $\varphi^{\alpha, \beta}$ implies that $-\left(\frac{|N|-1}{|N|}\right) \alpha \beta \geq 0$. So, $\alpha \beta \leq 0$ since $\frac{|N|-1}{|N|}>0$.

So, we conclude that $\alpha \beta=0$. Thus $\beta=\frac{1}{2}$ implies that $\alpha=0$. With Proposition 5.7 self-duality then implies that $\alpha=0$.

Note that by Theorem 4.4, instead of requiring the solution to belong to $\Phi$ we could also require that there exists a $\beta \in[0,1]$ such that the solution satisfies $\beta$-consistency.

Corollary 5.9 There exists a $\beta \in[0,1]$ such that solution $\psi$ satisfies $\beta$-consistency, 0 weak standardness for two-player games, efficiency, self-duality and is non-negative for non-negative games if and only if $\psi$ is the egalitarian solution.

We obtain the other self dual solutions (i.e., $\beta=\frac{1}{2}$ ) by requiring that payoffs react positively on a change in individual worth.

Proposition 5.10 Solution $\varphi^{\alpha, \beta} \in \Phi$ is self dual and reacts positive on a change in individual worth if and only if $\beta=\frac{1}{2}$.

\section{Proof}

Obviously the solutions $\varphi^{\alpha, \frac{1}{2}}, \alpha \in \mathbb{R}$, are self-dual and react positive on a change in individual worth. To show that these are the only solutions in $\Phi$ that react positive on a change in individual worth, take a null game $(N, v) \in \mathcal{G}$, i.e., $v(S)=0$ for all $S \subseteq N$. With (5.5) it follows that $\varphi_{i}^{\alpha, \beta}(N, v)=0$ for all $i \in N$.

Next, again take the standard game of some singleton, i.e. for some $i \in N$ take the game $(N, \bar{v})$ with $\bar{v}(\{i\})=1$, and $\bar{v}(S)=0$ for $S \neq\{i\}$. Again with (5.5) it follows that 
$\varphi_{i}^{\alpha, \beta}(N, v)=\left(1-\frac{1}{|N|}\right) \alpha \beta$. Since $\varphi^{\alpha, \beta}$ reacts positive on a change in individual worth, it must hold that $\left(1-\frac{1}{|N|}\right) \alpha \beta>0$, and thus $\alpha \beta>0$. With Proposition 5.7, self duality implies that $\beta=\frac{1}{2}$.

Again using Theorem 4.4, we can state the following corollary.

Corollary 5.11 There exists a $\beta \in[0,1]$ such that solution $\psi$ satisfies $\beta$-consistency, standardness for two-player games, efficiency, self-duality and reacts positive on a change in individual worth if and only if $\psi$ is equal to the average of the CIS-and ENSC-value $\varphi^{1, \frac{1}{2}}$.

The CIS-value is not self-dual. We can single out the CIS-value from the class $\Phi$ by requiring individual rationality on weakly essential games. Clearly, individual rationality and efficiency contradict if the game is not weakly essential.

Proposition 5.12 Consider solution $\varphi^{\alpha, \beta} \in \Phi$. Then $\varphi^{\alpha, \beta}$ is individually rational on weakly essential games if and only if it is the CIS-value $\varphi^{1,1}$.

PROOF

It is known that the CIS-value is individually rational for weakly essential games. To prove that it is the only solution in $\Phi$ that satisfies individual rationality, take a game $(N, v) \in \mathcal{G}$ with $|N| \geq 3$ such that for some $i \in N, v(\{i\})=v(N)=1$ and $v(S)=0$ otherwise. Then we have with (5.5) that

$$
\varphi_{i}^{\alpha, \beta}(N, v)=\alpha \beta+\frac{1}{|N|}-\frac{\alpha \beta}{|N|}=\left(1-\frac{1}{|N|}\right) \alpha \beta+\frac{1}{|N|} .
$$

Since $v$ is weakly essential, individual rationality of $\varphi^{\alpha, \beta}$ implies that $\left(1-\frac{1}{|N|}\right) \alpha \beta+\frac{1}{|N|} \geq 1$. Since $\left(1-\frac{1}{|N|}\right) \alpha \beta+\frac{1}{|N|} \geq 1 \Leftrightarrow\left(1-\frac{1}{|N|}\right)(\alpha \beta-1) \geq 0$, we conclude that $\alpha \beta \geq 1$ since $1>\frac{1}{|N|}$.

For $j \in N \backslash\{i\}$ we find with (5.5) that

$$
\varphi_{j}^{\alpha, \beta}(N, v)=\frac{1}{|N|}-\frac{\alpha \beta}{|N|}=\frac{1}{|N|}(1-\alpha \beta)
$$

Individual rationality of $\varphi^{\alpha, \beta}$ then implies that $\frac{1}{|N|}(1-\alpha \beta) \geq 0$. So, $\alpha \beta \leq 1$ since $\frac{1}{|N|}>0$. So, we conclude that $\alpha \beta=1$.

Next take a game $(N, \bar{v}) \in \mathcal{G}$ with $|N| \geq 3$ such that for some $i \in N, \bar{v}(N \backslash\{i\})=1$, and $\bar{v}(S)=0$ otherwise. With (5.5) it follows that

$$
\varphi_{i}^{\alpha, \beta}(N, v)=-\alpha(1-\beta)+\frac{1}{|N|} \alpha(1-\beta)=\alpha(1-\beta)\left(\frac{1}{|N|}-1\right) .
$$


Since $\bar{v}$ is weakly essential, individual rationality of $\varphi^{\alpha, \beta}$ implies that $\alpha(1-\beta)\left(\frac{1}{|N|}-1\right) \geq 0$. So, $\alpha(1-\beta) \leq 0$ since $\frac{1}{|N|}<1$.

For $j \in N \backslash\{i\}$ we find with (5.5) that

$$
\varphi_{j}^{\alpha, \beta}(N, v)=\frac{1}{|N|} \alpha(1-\beta) .
$$

Individual rationality of $\varphi^{\alpha, \beta}$ then implies that $\frac{1}{|N|} \alpha(1-\beta) \geq 0$, which is equivalent to $\alpha(1-\beta) \geq 0$.

So, we conclude that $\alpha(1-\beta)=0$.

Since we already showed the $\alpha \beta=1$ it must hold that $\alpha=1$, and thus also $\beta=1$.

Again using Theorem 4.4, we can state the following corollary.

Corollary 5.13 There exists a $\beta \in[0,1]$ such that solution $\psi$ satisfies $\beta$-consistency, standardness for two-player games, efficiency and individual rationality on weakly essential games if and only if $\psi$ is the CIS-value $\varphi^{1,1}$.

\section{Characterizations of $\Phi$ using weak individual ratio- nality}

In this section we again start by considering the whole class of solutions $\Phi$, and give a characterization of this class using a generalization of individual rationality for weakly essential games. First, for $\alpha \in \mathbb{R}$ and $\beta \in[0,1]$, a game $(N, v)$ is called $(\alpha, \beta)$-weakly essential if $v(N) \geq \sum_{i \in N} \lambda_{i}^{\alpha, \beta}(N, v)$ with $\lambda_{i}^{\alpha, \beta}(N, v)=\alpha(\beta v(\{i\})-(1-\beta) v(N \backslash\{i\}))$ as before.

Definition 6.1 A solution $\psi$ satisfies $(\alpha, \beta)$-individual rationality for $\alpha \in \mathbb{R}$ and $\beta \in$ $[0,1]$, if $\psi_{i}(N, v) \geq \lambda_{i}^{\alpha, \beta}(N, v)$ for every $(\alpha, \beta)$-weakly essential game $(N, v)$ and $i \in N$.

In van den Brink (2001) an axiomatic characterization of the Shapley value is given using efficiency, the null player property and fairness. Fairness states that if to a game $(N, v)$ we add a game $(N, w)$ in which players $i$ and $j$ are symmetric, then the payoffs of players $i$ and $j$ change by the same amount. Replacing the null player property by $(\alpha, \beta)$-individual rationality characterizes $\varphi^{\alpha, \beta}$. Although the solutions $\varphi^{\alpha, \beta}$ satisfy fairness, it is sufficient to require the weaker fairness property which states that the payoffs of all players change by the same amount if we only change the worth of the grand coalition. 
Definition 6.2 A solution $\psi$ satisfies weak fairness if and only if $(N, v),(N, w) \in \mathcal{G}$ satisfying $v(S)=w(S)$ for all $S \neq N$, implies that there is a $c^{*} \in \mathbb{R}$ such that

$$
\psi_{i}(N, w)-\psi_{i}(N, v)=c^{*} \text { for all } i \in N \text {. }
$$

Theorem 6.3 A solution $\psi$ satisfies efficiency, weak fairness and $(\alpha, \beta)$-individual rationality if and only if $\psi$ is equal to $\varphi^{\alpha, \beta}$.

PROOF

Obviously, $\varphi^{\alpha, \beta}$ satisfies the three properties. Now, suppose that solution $\psi$ satisfies efficiency, weak fairness and $(\alpha, \beta)$-individual rationality. For every $(N, v) \in \mathcal{G}$ define the game $(N, \bar{v})$ by $\bar{v}(S)=v(S)$ if $S \neq N$, and $\bar{v}(N)=\sum_{i \in N} \lambda_{i}^{\alpha, \beta}(N, v) . \quad(\alpha, \beta)$-individual rationality and efficiency imply that $\psi_{i}(N, \bar{v})=\lambda_{i}^{\alpha, \beta}(N, v)$ for all $i \in N$. Weak fairness implies that there is a $c^{*} \in \mathbb{R}$ such that $\psi_{i}(N, v)-\psi_{i}(N, \bar{v})=c^{*}$ for all $i \in N$. Efficiency then implies that $c^{*}=\frac{1}{|N|}(v(N)-\bar{v}(N))=\frac{1}{|N|}\left(v(N)-\sum_{j \in N} \lambda_{j}^{\alpha, \beta}(N, v)\right)$. Thus $\psi_{i}(N, v)=c^{*}+\psi_{i}(N, \bar{v})=\frac{1}{|N|}\left(v(N)-\sum_{j \in N} \lambda_{j}^{\alpha, \beta}(N, v)\right)+\lambda_{i}^{\alpha, \beta}(N, v)=\varphi_{i}^{\alpha, \beta}(N, v)$.

Similar as with weak standardness and weak consistency, we formulate weak individual rationality as follows.

Definition 6.4 A solution $\psi$ satisfies weak individual rationality if there exist $\alpha \in \mathbb{R}$ and $\beta \in[0,1]$ such that $\psi$ satisfies $(\alpha, \beta)$-individual rationality.

In a similar way as done with weak standardness and weak consistency, this property can be used to characterize the class of solutions $\Phi$.

Corollary 6.5 A solution $\psi$ satisfies efficiency, weak fairness and weak individual rationality, if and only if $\psi \in \Phi$.

Next we again discuss some specific solutions that we discussed before. For the special case $\alpha=\beta=1,(\alpha, \beta)$-individual rationality yields individual rationality for weakly essential games, and we obtain a characterization of the CIS-value.

Corollary 6.6 A solution $\psi$ satisfies efficiency, weak fairness and individual rationality for weakly essential games if and only if $\psi$ is equal to the CIS-value $\varphi^{1,1}$.

If $\alpha=0$ then $(\alpha, \beta)$-individual rationality states that all players earn non-negative payoffs whenever $v(N)$ is nonnegative, and we obtain a characterization of the egalitarian solution.

Corollary 6.7 A solution $\psi$ satisfies efficiency, weak fairness and non-negativity for games with non-negative worth of $N$ if and only if $\psi$ is equal to the egalitarian solution. 
In a similar way we characterize the solutions $\varphi^{\alpha, \beta}$ and the class $\Phi$ by replacing the null player property in the characterization of the Shapley value as given in Shapley (1953).

Theorem 6.8 A solution $\psi$ satisfies efficiency, symmetry, linearity and $(\alpha, \beta)$-individual rationality if and only if $\psi$ is equal to $\varphi^{\alpha, \beta} \in \Phi$.

\section{PROOF}

Obviously, $\varphi^{\alpha, \beta}$ satisfies the four properties. Now, suppose that solution $\psi$ satisfies efficiency, symmetry, linearity and $(\alpha, \beta)$-individual rationality. For every $(N, v) \in \mathcal{G}$ define the game $(N, \bar{v})$ as done in the proof of Theorem 6.3, i.e. $\bar{v}(S)=v(S)$ if $S \neq N$, and $\bar{v}(N)=$ $\sum_{i \in N} \lambda_{i}^{\alpha, \beta}(N, v)$. Further define the game $(N, \widehat{v})$ by $\widehat{v}(N)=v(N)-\sum_{j \in N} \lambda_{j}^{\alpha, \beta}(N, v)$, and $\widehat{v}(S)=0$ otherwise. Efficiency and $(\alpha, \beta)$-individual rationality again imply that $\psi_{i}(N, \bar{v})=$ $\lambda_{i}^{\alpha, \beta}(N, v)$. Efficiency and symmetry imply that $\psi_{i}(N, \widehat{v})=\frac{1}{|N|}\left(v(N)-\sum_{j \in N} \lambda_{j}^{\alpha, \beta}(N, v)\right)$ for all $i \in N$. Since $v=\bar{v}+\widehat{v}$, with additivity it follows that $\psi_{i}(N, v)=\psi_{i}(N, \bar{v})+\psi_{i}(N, \widehat{v})=$ $\lambda_{i}^{\alpha, \beta}(N, v)+\frac{1}{|N|}\left(v(N)-\sum_{j \in N} \lambda_{j}^{\alpha, \beta}(N, v)\right)=\varphi_{i}^{\alpha, \beta}(N, v)$.

Again, by taking specific values for $\alpha$ and $\beta$ we obtain characterizations of specific solutions ${ }^{4}$.

Corollary 6.9 A solution $\psi$ satisfies efficiency, symmetry, linearity and individual rationality for weakly essential games if and only if $\psi$ is equal to the CIS-value $\varphi^{1,1}$.

A solution $\psi$ satisfies efficiency, symmetry, linearity and non-negativity for games with non-negative worth of $N$ if and only if $\psi$ is equal to the egalitarian solution.

Finally, we have the following characterization of the class $\Phi$.

Corollary 6.10 A solution $\psi$ satisfies efficiency, symmetry, linearity and weak individual rationality if and only if $\psi \in \Phi$.

\section{Compromise values}

We conclude by looking at the solutions in $\Phi$ as compromise values. The literature on compromise values starts with the introduction of the $\tau$-value in Tijs (1981). This is an efficient solution for a special class of TU-games which is defined using the following two (non-efficient) solutions on $\mathcal{G}$. The marginal contribution (to the 'grand' coalition) is the solution $M^{\tau}$ given by $M_{i}^{\tau}(N, v)=v(N)-v(N \backslash\{i\})$ for all $i \in N$. The minimal right is

\footnotetext{
${ }^{4} \mathrm{An}$ alternative characterization of the egalitarian solution is given in van den Brink (2004).
} 
the solution $m^{\tau}$ given by $m_{i}^{\tau}(N, v)=\max _{\substack{S \subseteq N \\ i \in S}}\left(v(S)-\sum_{j \in S \backslash\{i\}} M_{j}^{\tau}(N, v)\right)$ for all $i \in N$. A game $(N, v) \in \mathcal{G}$ is called quasi-balanced if the following two conditions are satisfied

$$
m^{\tau}(N, v) \leq M^{\tau}(N, v) \text { and } \sum_{i \in N} m_{i}^{\tau}(N, v) \leq v(N) \leq \sum_{i \in N} M_{i}^{\tau}(N, v)
$$

The class of all quasi-balanced games is denoted by $Q B$. If $(N, v) \in Q B$ then the payoff vectors $m^{\tau}(N, v)$ and $M^{\tau}(N, v)$, respectively, can be seen as lower- and upper bounds for the distribution of $v(N)$ over the players in $N$. The $\tau$-value is the efficient solution on $Q B$ which assigns to every quasi-balanced game the unique efficient payoff vector on the line segment between $m^{\tau}(N, v)$ and $M^{\tau}(N, v)$, i.e., for every $(N, v) \in Q B$ it is given by

$$
\tau(N, v)=m^{\tau}(N, v)+\beta(v)\left(M^{\tau}(N, v)-m^{\tau}(N, v)\right)
$$

with

$$
\beta(v)=\left\{\begin{array}{cl}
\frac{v(N)-\sum_{i \in N} m_{i}^{\tau}(N, v)}{\sum_{i \in N}\left(M_{i}^{\tau}(N, v)-m_{i}^{\tau}(N, v)\right)} & \text { if } m^{\tau}(N, v) \neq M^{\tau}(N, v) \\
0 & \text { else. }
\end{array}\right.
$$

In Tijs (1987) it is shown that the $\tau$-value is the unique efficient solution on the class $Q B$ of quasi-balanced games that satisfies the minimal right property and restricted proportionality. Solution $\varphi$ satisfies

1. the minimal right property if for every $(N, v) \in Q B$ it holds that $\psi(N, v)=m^{\tau}(N, v)+$ $\psi\left(v-m^{\tau}(N, v)\right)$.

2. restricted proportionality if for every $(N, v) \in Q B_{0}:=\left\{(N, v) \in Q B \mid m^{\tau}(N, v)=0\right\}$ the vector $\psi(N, v) \in \mathbb{R}^{n}$ is proportional to the vector $M^{\tau}(N, v) \in \mathbb{R}^{n}$.

Note that the marginal contribution and minimal right vectors that are used in the definition of the $\tau$-value appear in the two properties that characterize the $\tau$-value. The minimal right property can be replaced by the property of Covariance ${ }^{5}$ which is stronger than the minimal right property but does not use the specific minimal right vector. But the minimal right and marginal contribution vector still appear in the restricted proportionality property.

A disadvantage of the $\tau$-value is that it does not exist for games that are not quasibalanced. For these games the marginal contribution and minimal right are inadequate as upper- and lower bounds for the distribution of payoffs. But for such games other bounds

\footnotetext{
${ }^{5}$ The solution $\psi$, satisfies the Covariance property on $\mathcal{C} \subseteq \mathcal{G}$ if $\psi(N, k v+c)=k \psi(N, v)+c$ for all $(N, v) \in \mathcal{C}, k \in \mathbb{R}_{+}$and $c \in \mathbb{R}^{n}$ such that $(N, k v+c) \in \mathcal{C}$, where the game $(N, k v+c)$ is given by $(k v+c)(S)=k v(S)+\sum_{i \in S} c_{i}$.
} 
can be appropriate yielding alternative compromise values. If we take two solutions $m$ and $M$ such that for game $(N, v)$ the conditions stated under (7.6) are satisfied with $m^{\tau}$ and $M^{\tau}$ replaced by $m$ and $M$, respectively, then the values $m(N, v)$ and $M(N, v)$ can be seen as lower- and upper bounds for the distribution of payoffs in game $(N, v)$. Denoting by $\mathcal{G}(m, M) \subset \mathcal{G}$ the class of games for which the conditions under (7.6) are satisfied in terms of $m$ and $M$, we define $t^{(m, M)}$ as the solution which assigns to every $(N, v) \in \mathcal{G}(m, M)$ the unique efficient payoff vector on the line segment between $m(N, v)$ and $M(N, v)$. (Thus $\tau=t^{\left(m^{\tau}, M^{\tau}\right)}$.) If $m$ and $M$ satisfy covariance then $t^{(m, M)}$ can be axiomatized similarly as the $\tau$-value by replacing $m^{\tau}$ and $M^{\tau}$ in the minimal right and restricted proportionality properties by $m$ and $M$, respectively. (The proof of this result is similar to the proof of the characterization of the $\tau$-value as given in Tijs $(1987)^{6}$.)

By taking as lower- and upper bounds the solutions $m^{\alpha, \beta}$ and $M^{\alpha, \beta}$ given by

$$
m_{i}^{\alpha, \beta}(N, v)=\lambda_{i}^{\alpha, \beta}(N, v)
$$

and

$$
M_{i}^{\alpha, \beta}(N, v)=v(N)-\sum_{j \in N \backslash\{i\}} m_{j}^{\alpha, \beta}(N, v)=v(N)-\sum_{j \in N \backslash\{i\}} \lambda_{j}^{\alpha, \beta}(N, v) \text { for all } i \in N,
$$

we obtain the solution $t^{\left(m^{\alpha, \beta}, M^{\alpha, \beta}\right)}$ which assigns to every $(N, v) \in \mathcal{G}\left(m^{\alpha, \beta}, M^{\alpha, \beta}\right)$ the unique efficient payoff vector on the line segment between $m^{\alpha, \beta}$ and $M^{\alpha, \beta}$. This solution is equal to $\varphi^{\alpha, \beta} \in \Phi$. Since $m^{\alpha, \beta}$ and $M^{\alpha, \beta}$ both satisfy covariance, $\psi^{\alpha, \beta} \in \Phi$ is characterized by efficiency, covariance (or the minimal right property) and restricted proportionality stated with the appropriate bounds. Solution $\varphi$ satisfies

1. the minimal right property with respect to $m^{\alpha, \beta}$ if for every $(N, v) \in Q B$ it holds that $\psi(N, v)=m^{\alpha, \beta}(N, v)+\psi\left(v-m^{\alpha, \beta}(N, v)\right)$.

2. restricted proportionality with respect to $m^{\alpha, \beta}$ and $M^{\alpha, \beta}$ if for every $(N, v) \in Q B_{0}:=$ $\left\{(N, v) \in Q B \mid m^{\alpha, \beta}(N, v)=0\right\}$ the vector $\psi(N, v) \in \mathbb{R}^{n}$ is proportional to the vector $M^{\alpha, \beta}(N, v) \in \mathbb{R}^{n}$.

Corollary 7.1 The solution $\varphi^{\alpha, \beta}$ is the unique solution $\psi$ on $\mathcal{G}\left(m^{\alpha, \beta}, M^{\alpha, \beta}\right)$ that satisfies efficiency, the minimal right property with respect to $m^{\alpha, \beta}$ and restricted proportionality with respect to $m^{\alpha, \beta}$ and $M^{\alpha, \beta}$.

\footnotetext{
${ }^{6}$ In that proof only twice use is made of the specific formula's of $m^{\tau}(N, v)$ and $M^{\tau}(N, v)$. This is to show that for every $v \in \mathcal{G}, m^{\tau}\left(v-m^{\tau}(N, v)\right)$ is the nullvector (which is satisfied for all $m$ satsifying covariance), and to show that $M^{\tau}$ satisfies covariance.
} 


\section{Concluding remarks}

In this paper we defined a class of solutions for two-player games satisfying $\alpha$-standardness for some $\alpha \in \mathbb{R}$, and extended this to $n$-player games using $\beta$-consistency, $\beta \in[0,1]$. Within this class every solution is characterized by values of the parameters $\alpha \in \mathbb{R}$ and $\beta \in[0,1]$. Note that the parameter $\beta$ does not appear in the standardness property, while the parameter $\alpha$ does not appear in the consistency property. Basically, the parameter $\alpha$ is some measure of equity. The closer $\alpha$ is to zero, the closer our solution is to the egalitarian solution. For a given equity measure $\alpha$, the parameter $\beta \in[0,1]$ determines to what extent we use the individual worths $v(\{i\})$ or the marginal contributions $v(N)-v(N \backslash\{i\})$ in defining our solution. This difference is also reflected in the reduced games.

We once more remark that for every solution $\varphi^{\alpha, \beta} \in \Phi$ the values of the parameters are fixed and are the same for every game. We obtain a larger class of solutions if we allow these parameters to depend on the game. Allowing the value of $\alpha$ to depend on the game we could take $\alpha(v)=\frac{v(N)}{v(\{i\})+v(\{j\})}$ whenever $v(\{i\})+v(\{j\}) \neq 0$. This yields proportional standardness for two-player games as satisfied by, e.g. the Proper Shapley value as introduced in Vorob'ev and Liapunov (1998): for $N=\{i, j\}, i \neq j$, we have $\psi_{i}(N, v)=$ $\frac{v(N)}{2(v(\{i\})+v(\{j\}))}\left(v(\{i\}-v(\{j\}))+\frac{1}{2} v(N)=\frac{v(\{i\})-v(\{j\})+v(\{i\})+v(\{j\})}{2(v(\{i\}+v(\{j\})} v(N)=\frac{v(\{i\})}{v(\{i\})+v(\{j\})} v(N)\right.$. In van den Brink and Funaki (2004) it is shown that the $\tau$-value, although not a solution in $\Phi$, for convex games always is a convex combination of the CIS- and ENSC-value with weights determined by the parameter $\beta$. However, for different games the weight assigned to these two solutions is different. So, allowing the parameter $\beta(v)$ to depend on the game we also consider the $\tau$-value when we restrict ourselves to convex games ${ }^{7}$. In the future we study the generalized class of solutions with parameters $\alpha$ and $\beta$ depending on the game, and in that way obtaining new characterizations of (solutions related to) the $\tau$-value and the Proper Shapley value.

Given our class of solutions $\Phi$ we can also define a new set-valued solution for TUgames by assigning to every game the union of all payoff vectors assigned by any solution in $\Phi$ to that game, i.e. we can consider the set-valued solution $\Psi$ given by $\Psi(N, v)=$ $\left\{\varphi^{\alpha, \beta}(N, v) \mid \alpha \in \mathbb{R}, \beta \in[0,1]\right\}$. Finding axiomatic characterizations for this set-valued solution is a plan for future research. Further, the solutions considered in Joosten (1996) and Ju, Borm and Ruys (2004) who, respectively, consider all convex combinations of the egalitarian solution and the Shapley value, and all convex combinations of the CIS-value and the Shapley value, can be generalized by considering all convex combinations of any solution $\varphi^{\alpha, \beta} \in \Phi$ and the Shapley value.

Finally, we mention that it is interesting to find an implementation of the solutions

\footnotetext{
${ }^{7}$ The weight parameter $\beta(v)$ is exactly given by (7.7).
} 
in $\Phi$.

\section{Appendix}

As mentioned in Section 4, it is not necessary to have the parameter $\beta$ enter the reduced game for more than three player games. If $|N| \geq 4$ we could take any $\beta \in[0,1]$ to obtain a characterization of the solutions in $\Phi^{\beta}$. However, it is crucial that the parameter $\beta$ appears in the reduced game for three player games. Besides the reduced game defined in this paper we could also use other reduced games. An illustration of such a reduced game is the following.

Take a game $(N, v)$, a payoff vector $x$, and a player $j$. The player set of a reduced game again is obtained by removing player $j$ from the original player set $N$. The worth of the coalition $N \backslash\{j\}$ is equal to the worth of $N$ minus the payoff $x_{j}$. In the case $|N| \geq 4$, the worth of a proper subcoalition with $|S| \leq|N|-3$ in $N \backslash\{j\}$ is equal to the same amount as in the original game and the worth of a proper subcoalition with $|S|=|N|-2$ in $N \backslash\{j\}$ is equal to the worth of $S \cup\{j\}$. In the case $|N|=3$, the worth of a proper subcoalition (one-player coalition) is a convex combination of the worth of the coalition and the union of the coalition and the removing player for the original game.

Definition 8.1 Take $\beta \in[0,1]$. Given a game $(N, v) \in \mathcal{C}$ with $|N| \geq 3$, a player $j \in N$, and a payoff vector $x \in \mathbb{R}^{N}$, the $\beta$-reduced game with respect to $j$ and $x$ is the game $\left(N \backslash\{j\}, v^{x}\right)$ where, for the case $|N| \geq 4$,

$$
v^{x}(S)= \begin{cases}v(N)-x_{j} & \text { if } S=N \backslash\{j\} \\ v(S \cup\{j\}) & \text { if } S \subset N \backslash\{j\},|S|=|N|-2 \\ v(S) & \text { if } S \subset N \backslash\{j\},|S| \leq|N|-3 .\end{cases}
$$

and, for the case $|N|=3$,

$$
v^{x}(S)= \begin{cases}v(N)-x_{j} & \text { if } S=N \backslash\{j\} \\ \beta v(S)+(1-\beta) v(S \cup\{j\}) & \text { if } S \subset N \backslash\{j\}, S \neq \emptyset, \\ 0 & \text { if } S=\emptyset .\end{cases}
$$

Indeed, we need $v^{x}(S)=v(S)$ for $|S|=1$ and $v^{x}(S)=v(S \cup\{j\})$ for $|S|=|N|-2$. For other coalitions $S$ with $2 \leq|S| \leq|N|-3$, we can define any value for the worth of those coalitions by some consistent way, such that the order of removing players from the original set has no effect on the worth. However the first two definitions are inconsistent for the case of $|N|=3$. Then we need a different definition: $v^{x}(S)=\beta v(S)+(1-\beta) v(S \cup\{j\})$, 
which depends on a coefficient $\beta$. This $\beta$ is related to our value $\varphi^{\alpha, \beta}$ of $n$-player games. The reduced game for $|N| \geq 4$ is similar to the reduced games considered in Funaki and Yamato (2001). But they use $v(S \cup\{j\})-x_{j}$ instead of $v(S \cup\{j\})$.

$\beta$-consistency now can be defined in a similar way as in Section 5 , but taking the reduced game from Definition 8.1 instead of the reduced game of Definition 4.1. Similar as Theorem 4.4 in the main text we can show the following theorem.

Theorem 8.2 Take any $\beta \in[0,1]$ and $\alpha \in \mathbb{R}$. A solution $\psi$ satisfies efficiency, $\beta$ consistency with respect to the reduced game of Definition 8.1 and $\alpha$-standardness for twoplayer games on the class of all games $\mathcal{G}$, if and only if $\psi=\varphi^{\alpha, \beta}$.

In this way all the solutions in $\Phi$ satisfy the same conistency for more than three-player games, but for the different $\beta \in[0,1]$ the solutions in $\Phi^{\beta}$ are characterized by the different consistency properties with different $\beta$ for three-player games. Similar as in Section 5 we obtain characterization of specific solutions from $\Phi$ as corollaries.

\section{References}

Brink, R. van den (2001) "An Axiomatization of the Shapley Value using a Fairness Property", International Journal of Game Theory, 30, 309-319.

Brink, R. van den (2004) "Null Players or Zero Players: The Difference Between the Shapley Value and the Egalitarian Solution", Discussion paper TI 2004-127/1, Tinbergen Institute, Amsterdam/Rotterdam, The Netherlands.

Brink, R. van den and Y. Funaki (2004) "The Equal Surplus Sharing Set for TU-Games", Mimeo.

Driessen, T.S.H., and Y. Funaki (1991) "Coincidence of and Collinearity between Game Theoretic Solutions", OR Spektrum, 13, 15-30.

Funaki, Y. (1998) "Dual Axiomatizations of Solutions of Cooperative Games", Mimeo.

Funaki, Y. and T. Yamato (2001) "The Core and Consistency Properties: A General Characterization", International Game Theory Review, 3, 175-187.

Hart, S., and A. Mas-Colell (1988), "The Potential of the Shapley Value", in: The Shapley Value. Essays in Honor of L.S. Shapley (ed. A.E. Roth), Cambridge University Press, pp. 127-137. 
Hart, S., and A. Mas-Colell , (1989), "Potential, Value and Consistency", Econometrica, 57, 589-614.

Joosten, R. (1996), Dynamics, Equilibria and Values, Dissertation, Maastricht University.

Ju, Y., P.E.M. Borm, and P.H.M. Ruys (2004), "The consensus value : a new solution concept for cooperative games", Center Discussion paper 2004-50, Tilburg University, The Netherlands.

Shapley, L.S. (1953) "A Value for $n$-Person Games", in: Annals of Mathematics Studies 28 (Contributions to the Theory of Games Vol.2) (eds. H.W. Kuhn and A.W. Tucker), Princeton University Press, pp.307-317.

Tijs, S.H. (1981) "Bounds for the Core and the $\tau$-value", in O.Moeschlin and D. Pallaschke (eds.), Game Theory and Mathematical Economics, North-Holland Publishing Company, 123-132, Amsterdam.

Tijs, S.H. (1987) "An Axiomatization of the $\tau$-value", Mathematical Social Sciences, 13, $177-181$.

Vorob'ev and Liapunov (1998), "The Proper Shapley Value", In Game Theory and Applications Vol IV (L.Petrosjan and M. Mazalov eds), Nova Science, New York, pp. 155-159.

Weber, R.J. (1988), "Probabilistic Values for Games", in A.E. Roth (editor), The Shapley Value: Essays in Honor of Lloyd S. Shapley, Cambridge University Press, Cambridge, MA. 